\title{
Microstructure of a Ti-50 wt\% Ta alloy produced via laser powder bed fusion
}

\author{
Lei-Lei Xing ${ }^{1} \cdot$ Cong-Cong Zhao ${ }^{2} \cdot$ Hao Chen ${ }^{1} \cdot$ Zhi-Jian Shen $^{1,3} \cdot$ Wei Liu ${ }^{1}$
}

Received: 18 October 2019 / Revised: 26 November 2019 / Published online: 8 May 2020

(c) The Chinese Society for Metals (CSM) and Springer-Verlag GmbH Germany, part of Springer Nature 2020

\begin{abstract}
Ti-Ta alloys have been widely studied for biomedical applications due to their high biocompatibility and corrosion resistance. In this work, nearly fully dense and in situ alloyed Ti-50 wt $\%$ Ta samples were fabricated by the laser powder bed fusion (LPBF) of mechanically mixed powders. With increased exposure time, and thereby increased laser energy density, insoluble Ta particles were almost dissolved, and a Ti-50 wt\% Ta alloy was formed. Cellular and dendritic structures were formed due to constitutional undercooling, which was caused by the high cooling rate of LPBF process. Both retained $\beta$ phases and $\alpha^{\prime \prime}$ phases were observed in the LPBFed Ti-50 wt \% Ta alloy. The $\alpha^{\prime \prime}$ phase was found at the boundary of the cellular structures, where the tantalum content was not high enough to suppress the bcc lattice transition completely but could suppress the $\beta$ phase $\rightarrow \alpha^{\prime}$ phase transition.
\end{abstract}

Keywords Laser powder bed fusion · Ti-Ta alloys $\cdot$ Cellular and dendritic structures $\cdot$ Microsegregation $\cdot$ Phase transformation

\section{Introduction}

The American Society for Testing Materials (ASTM) has defined additive manufacturing (AM) as "a process of joining materials to make objects from three-dimensional (3D) model data, usually layer upon layer, as opposed to subtractive manufacturing methodologies"' [1]. Laser powder bed fusion (LPBF) is a member of the additive manufacturing family, in which a 3D part is built by laser scanning a precursor powder bed [2]. LPBF possesses several advantages over other production techniques, such as high material use efficiency, a high level of flexibility, and near net shape

Available online at http://link.springer.com/journal/40195.

Wei Liu

liuw@mail.tsinghua.edu.cn

1 School of Materials Science and Engineering, Tsinghua University, Beijing 100084, China

2 Jihua Laboratory, 13th Floor, Chengye building, Nanping West Road No. 13, Guicheng, Nanhai district, Foshan city, Guangdong province 528000, China

3 Arrhenius Laboratory, Department of Materials and Environmental Chemistry, Stockholm University, 106 91, Stockholm, Sweden production of geometrically complex structural parts [3]. Furthermore, this technology can decrease element segregation [4] and grain growth because of the high cooling rates $\left(>10^{3}{ }^{\circ} \mathrm{C} / \mathrm{s}\right)[5]$.

Titanium alloys, especially Ti-6Al-4V, are widely used for aerospace and medical applications due to their excellent specific strength, toughness, corrosion resistance, and biocompatibility [5-8]. However, conventional manufacturing processes of titanium products always cause a large amount of material waste, have high manufacturing costs, and are time intensive. To mitigate these drawbacks, LPBF techniques have been applied extensively in the manufacturing of titanium alloys with complex structures. Recently, safety concerns about how alloy elements effect the human body have been received more attention [9-12]. When compared with vanadium and aluminum, tantalum alloys have been investigated by many researchers due to its superior corrosion resistance, better biocompatibility, and higher strength [13-18]. Moreover, Ti-Ta alloys have a lower elastic modulus than other titanium alloys, with a value closer to that of human bone. The microstructure and property of Ti-Ta alloys change with different Ta contents. Among these alloys, Ti-50 wt\% Ta alloy was received considerable attention for its outstanding corrosion resistance [19]. 
Titanium alloys, which are generally multicomponent alloys containing $\alpha$-stabilizing as well as $\beta$-stabilizing elements, are broadly classified as $\alpha$ alloys, $\alpha+\beta$ alloys and $\beta$ alloys. In $\beta$ alloys, the $\beta$-phase is stabilized by the addition of adequate amounts of $\beta$ stabilizing elements and can be retained at room temperature [20]. In addition to these equilibrium phases, non-equilibrium phases, especially martensite [21], will form during the process of rapid cooling from the $\beta$ phase field. The martensite phases include $\alpha^{\prime}$ phase and $\alpha^{\prime \prime}$ phase, which depends on the content of $\beta$-stabilizing elements. Ta, as a $\beta$-stabilizing element, suppresses the transformation from the $\beta$ phase with bodycentered cubic structure to the $\alpha^{\prime}$ phase with hexagonal close-packed structure. Within a certain content range of $\beta$-stabilizing elements, the metastable $\alpha^{\prime \prime}$ phase with the orthorhombic structure (the transition lattice of body-centered cubic lattice to hexagonal close-packed lattice) appears during rapid cooling. Some researchers [22] have reported the phase compositions of Ti-Ta alloys with increasing $\mathrm{Ta}$ contents by using regular forming methods. They found $\alpha^{\prime}$ phase formed at $20 \mathrm{wt} \%$ Ta content, the $\alpha^{\prime \prime}$ phase formed at $40-50 \mathrm{wt} \%$ Ta content, the $\alpha^{\prime \prime}+\beta$ phase formed at $60 \mathrm{wt} \% \mathrm{Ta}$ content, and $\beta$ phase only at $70-80 \mathrm{wt} \%$ Ta content.

It is difficult and expensive to fabricate Ti-Ta alloys by using regular forming methods due to the substantial density difference between $\mathrm{Ti}\left(4.54 \mathrm{~g} / \mathrm{cm}^{3}\right)$ and $\mathrm{Ta}\left(16.6 \mathrm{~g} / \mathrm{cm}^{3}\right)$, which leads to inhomogeneity and segregation of elements during the solidification process. To avoid the segregation of elements, the alloy ingots must be remelted many times with flipping between two melting processes and homogenization in vacuum at high temperature for long periods of time [23]. Sing et al. [4] manufactured Ti-Ta parts that consisted of equiaxed $\beta$ grains and laminar substructure via LPBF initially. In their report [24], the influence of processing parameters on the microstructure and properties such as relative density, microhardness, and surface roughness was investigated. Furthermore, they found the dimensional accuracy and mechanical properties of the cellular lattice structures are sensitive to the processing parameters [25]. Zhao et al. [26] studied the microstructure evolution, mechanical properties by adjusting the content of Ta. Researchers have made tremendous efforts for the preparation of TiTa alloys via LPBF. However, the detailed investigation into the microstructure such as cellular/dendritic features and phase transformation is still limited. And it is extremely important to make out the TiTa alloys formation via LPBF, which was different from the microstructure manufactured by arc melting.

TiTa alloy powder is difficult and expensive to fabricate due to the complicated and time-consuming process. Furthermore, the TiTa alloy ingot for producing powder is difficult to achieve the homogenization of elements, which is caused by the large density difference of Ti $(4.54 \mathrm{~g} /$ $\left.\mathrm{cm}^{3}\right)$ and Ta $\left(16.6 \mathrm{~g} / \mathrm{cm}^{3}\right)$. However, in situ alloyed Ti-50 wt\% Ta samples can be fabricated by the laser powder bed fusion (LPBF) by using mechanically mixed powders. In this paper, a Ti-50 wt \% Ta alloy with good metallurgical microstructure and minimal defects was fabricated by LPBF with different process parameters. The situ alloying, microstructure, component distribution, and phase composition were investigated. This study provides a new method for controlling microstructures with different phase distributions in a fine cellular structure via laser powder bed fusion.

\section{Materials and methods}

\subsection{Powder preparation}

The nominal Ti-50 wt $\%$ Ta powder was prepared using Ti (Grade 1, TLS Technology, Germany, purity $>99.5 \%$, size $<53 \mu \mathrm{m}$ ) and Ta (Metallurgical grade, Ningxia Orient Tantalum Industry, China, purity $>99.5 \%$, size $<20 \mu \mathrm{m}$ ) commercial powders. The powders were mixed in ethanol using a tumbler mixer for $6 \mathrm{~h}$. The mixed powder was then dried in a vacuum drying chamber for $12 \mathrm{~h}$ to remove the ethanol.

\subsection{Laser powder bed fusion experiments}

All the samples were fabricated on pure titanium substrates by using a Renishaw AM 250 machine (Renishaw, UK). The machine was equipped with a $200 \mathrm{~W}$ Yb:YAG fiber laser with a spot size of $75 \mu \mathrm{m}$. The laser source was used in pulse mode, meaning that the laser focused on one point for a given period ( $T$, exposure time) at a given power $(P$, power) and then moved to the next point to repeat the exposure process. The distance between two points was defined as the point distance (pd). The distance between parallel lines was defined as the hatch spacing $(h)$. Layers were scanned using a zigzag pattern in which a $67^{\circ}$ rotation was used in between each layer, as illustrated in Fig. 1. The powder layer thickness was represented as $t$.

Because of the high melting point of $\mathrm{Ta}\left(2996{ }^{\circ} \mathrm{C}\right.$, which is much higher than that of Ti), the influence of the exposure time on the situ alloying of the Ti-50 wt\% Ta alloy was considered. Other parameters were set as constant values. The LPBF processing parameters for all the specimens are listed in Table 1. The samples fabricated with different parameters were labeled as S1, S2, S3, S4, respectively. Multiple cube specimens with dimensions of $10 \mathrm{~mm} \times 10 \mathrm{~mm} \times 10 \mathrm{~mm}$ were fabricated. Argon was used to ensure that the oxygen content was below $500 \mathrm{ppm}$. The 
$67^{\circ}$ Rotation

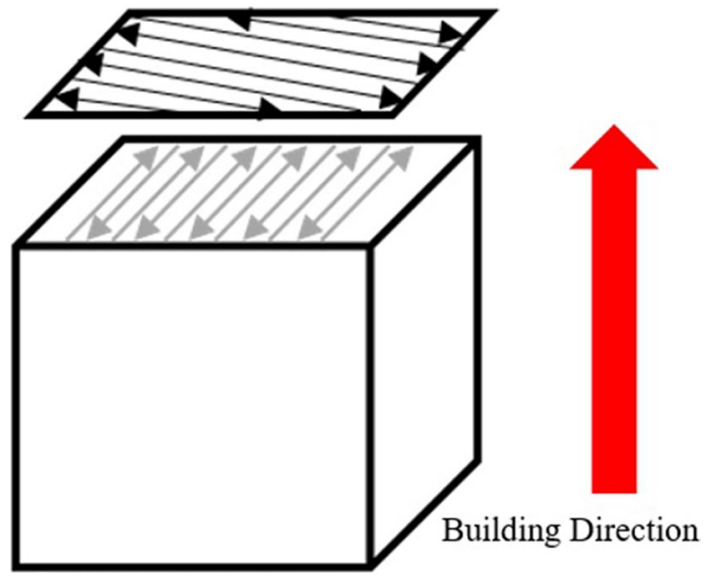

Fig. 1 Laser scanning schematic diagram

laser energy density $(E)$ was also calculated and is shown in Table 1; this value was obtained by using the following equation: $E=\frac{P \times T}{t \times \mathrm{pd} \times h}$.

\subsection{Microstructural characterization}

The composition of the mixed powders was measured using X-ray fluorescence (XRF, Thermo Fisher ARL PERFORM X). The microstructures of the powders and LPBFed samples were observed by a scanning electron microscope (SEM, TESCAN) equipped with an electron backscatter diffraction detector (EBSD, Oxford Instruments, Oxford, UK) and an energy-dispersive spectrometer (EDS). The samples for EBSD analysis were electrically polished with a solution of $97 \%$ ethanol and $3 \%$ perchloric acid. Phase identification was performed by using X-ray diffraction (XRD, D/Max $2500 \mathrm{~V}$ ) with a $\mathrm{Cu}$ target in a conventional X-ray tube operating at an accelerating voltage of $40 \mathrm{kV}$ and an electron beam current of $30 \mathrm{~mA}$. The scattering angle $(2 \theta)$ varied from $30^{\circ}$ to $90^{\circ}$ with a scan rate of $10^{\circ}$ per minute. The finer microstructure was further characterized by transmission electron microscope (TEM, JEOL-2100) operating at $200 \mathrm{kV}$. The TEM samples were prepared by grinding a $3 \mathrm{~mm}$ diameter disk to approximately $30 \mu \mathrm{m}$ and then finally thinned by a Gatan-made precision ion polishing system (PIPS) at $5 \mathrm{keV}$ with a gun angle of $\pm 8^{\circ}$.

\section{Results and discussion}

SEM images of initial $\mathrm{Ti}$ and Ta powders are presented in Fig. 2a, b. The titanium powder had a spherical morphology with an average diameter of 30-34 $\mu \mathrm{m}$. The tantalum powder particles were irregularly shaped with an average diameter of 15-20 $\mu \mathrm{m}$. The average diameter of Ta powder is smaller than the LPBFed powders, which would be beneficial to the melting of Ta. It can be clearly seen that the Ta powder consisted of a higher fraction of small size particles than the Ti powder. The morphology and EDS results of the mixed Ti-50 wt\% Ta powders are shown in Fig. 2c, d. It is clear that the powders were mixed very evenly, and no agglomeration occurred. The size and shape of the spherical titanium powder and non-spherical tantalum powder did not change after mixing. The EDS result indicated that the weight percent of $\mathrm{Ti}$ and $\mathrm{Ta}$ in the mixed powders was $53.5 \mathrm{wt} \%$ and $46.5 \mathrm{wt} \%$, respectively. This result was very close to the XRF results, as shown in Table 2, and the design composition, suggesting that this method is appropriate for mixing the powders. The term Ti-50 wt $\%$ Ta is used throughout the article.

The mixed powder was used to fabricate $\mathrm{Ti}-50 \mathrm{wt} \%$ Ta alloys by LPBF with different parameters, as shown in Table 1. Figure $3 \mathrm{a}-\mathrm{d}$ shows the microstructures of the cubic S1-S4 samples. Numerous white regions with irregular shape were clearly observed, which were confirmed to be Ta particles by EDS. The other regions are Ti-Ta solid solutions, as shown by the EDS results in the inset of Fig. $3 \mathrm{~d}$. It was clearly demonstrated that the size and the proportion of white regions decreased noticeably when the exposure time was increased. The variation in the proportion of unmelted Ta can be counted quantitatively through statistical analysis of a large number of SEM images. As shown in Fig. 3e, the percentage of unmelted Ta was $11 \%$ at $50 \mu$ s of exposure time and then decreased to $7.1 \%$ at $150 \mu \mathrm{s}$. When the exposure time increased to $500 \mu \mathrm{s}$ (S4 sample), the percentage was significantly reduced to $1.7 \%$, and the contents of $\mathrm{Ti}$ and Ta are almost equal and homogeneous. Therefore, increasing the laser energy density is beneficial in the fabrication of $\mathrm{Ti}-\mathrm{Ta}$ alloys using this processing method. Some researchers $[27,28]$ proposed a model to describe the in situ alloying processing for particles, which included two parameters.
Table 1 LPBF processing parameters for $\mathrm{Ti}-50 \mathrm{wt} \% \mathrm{Ta}$

\begin{tabular}{llllllc}
\hline Sample & $P(\mathrm{~W})$ & $t(\mu \mathrm{m})$ & $\mathrm{pd}(\mu \mathrm{m})$ & $T(\mu \mathrm{s})$ & $h(\mu \mathrm{m})$ & $E\left(\mathrm{~J} / \mathrm{mm}^{3}\right)$ \\
\hline S1 & 200 & 30 & 40 & 80 & 80 & 167 \\
S2 & 200 & 30 & 40 & 150 & 80 & 313 \\
S3 & 200 & 30 & 40 & 300 & 80 & 625 \\
S4 & 200 & 30 & 40 & 500 & 80 & 1042 \\
\hline
\end{tabular}



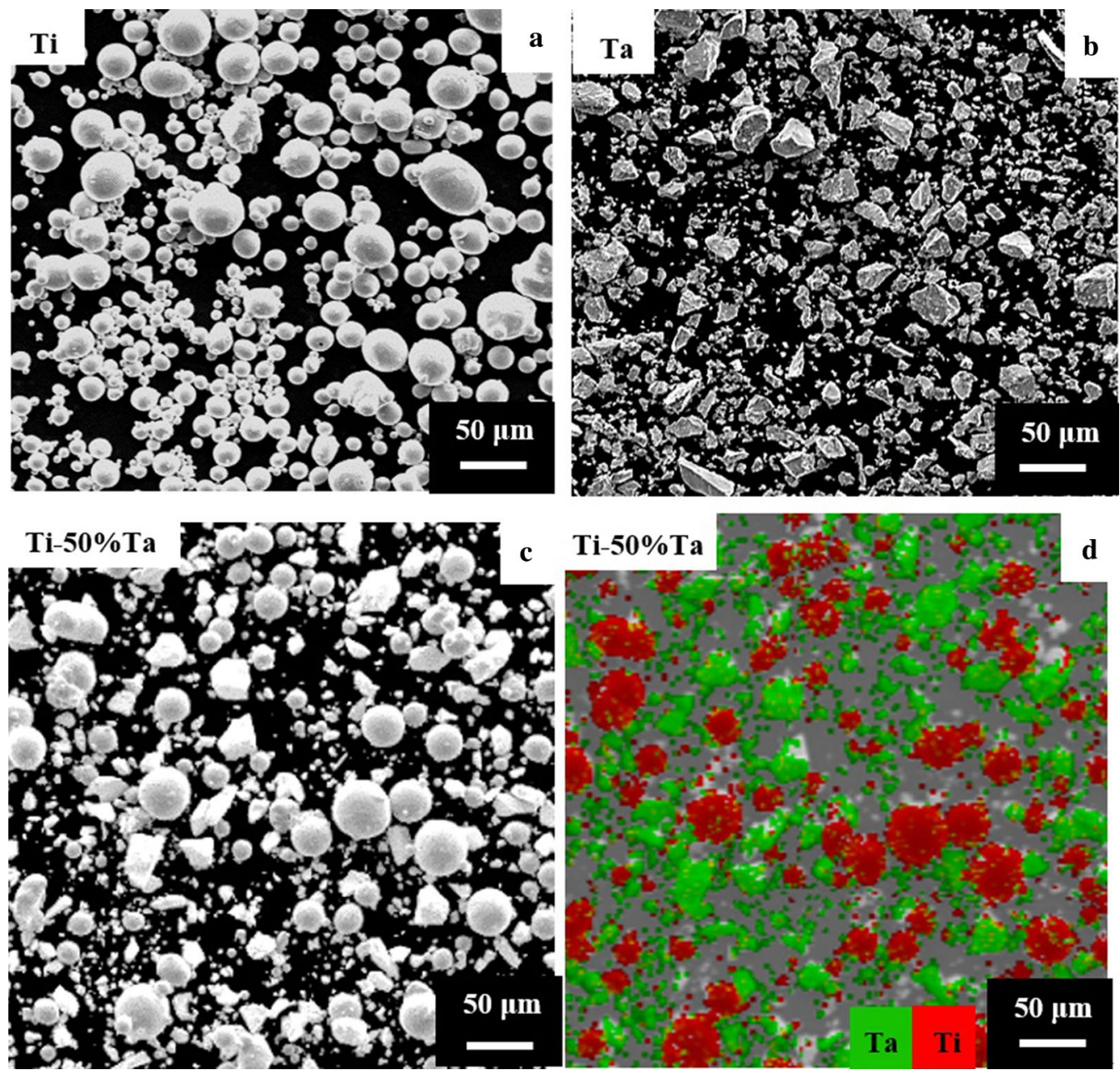

Fig. 2 SEM and EDS micrographs for a pure titanium powder; $\mathbf{b}$ pure tantalum powder; $\mathbf{c}$ the Ti-50 wt\% Ta powder; $\mathbf{d}$ EDS results corresponding to image $\mathbf{c}$

Table 2 Element content at points A, B, and C in Fig. 4d

\begin{tabular}{lll}
\hline Point & $\mathrm{Ti}(\mathrm{wt} \%)$ & $\mathrm{Ta}(\mathrm{wt} \%)$ \\
\hline A & 60.8 & 39.2 \\
B & 53.3 & 46.7 \\
C & 41.5 & 58.5 \\
\hline
\end{tabular}

One parameter is the energy required to melt the particles, called E.

$E=\rho\left[C \times\left(T_{\mathrm{m}}-T_{0}\right)+L_{\mathrm{f}}\right]$

where $\rho$ is the density of the particles, $C$ is the heat capacity, $T_{\mathrm{m}}$ is the melting point, and $L_{\mathrm{f}}$ is the latent heat of fusion. The other parameter is the time required for heat to transfer to the particle core, called $t_{\text {hom}}$.

$t_{\text {hom }}=r^{2} / k_{\mathrm{b}}$, where $r$ is the powder particle radius and $k_{\mathrm{b}}$ is the thermal diffusivity. For the particles to which was made of alloy, the laser energy should be higher than $E$, and laser exposure time should be higher than $t_{\text {hom }}$. It was calculated that the energy required for melting Ta is $9.2515 \mathrm{~J} / \mathrm{mm}^{3}$, and the time required for homogenization is $16.4 \mu \mathrm{s}$. It is much lower than all LPBF processing parameters. It is influenced by the laser absorptivity of Ta particles, laser's working state as well as the molten pool convection and Marangoni flows. However, it can be concluded that increasing the exposure time is very effective for reducing the unmelted Ta and achieving homogeneity of the elements. It should be pointed out that increasing the exposure time should be controlled in a range [29]. With the endless increasing in exposure time, the time of solidification will increase, and it will aggravate the element aggregation [30]. It would be investigated detailedly in our future work.

A large number of experiments observation have confirmed that large forming defect was inexistent, and few 

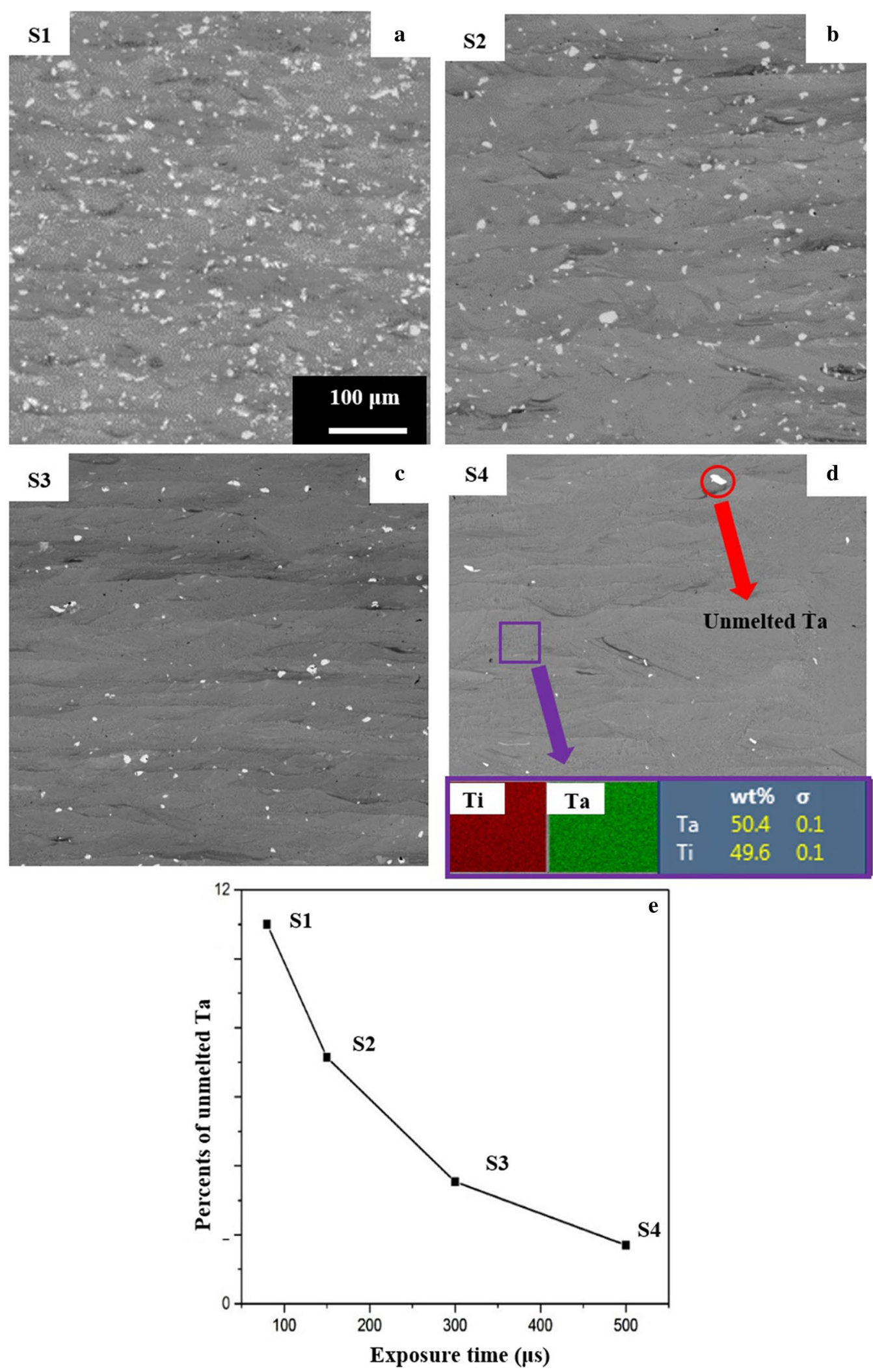

Fig. 3 a-d Backscattered electron (BSE) micrographs of the cubic S1-S4 samples; e percent of unmelted Ta for S1-S4 samples 
pores were found in all the fabricated samples which revealed the samples were fully solid with a relative density near $100 \%$. It can be seen that sample S4, which consisted of a Ti-Ta solution matrix with slight unmelted tantalum particles, had the best situ alloying result. Therefore, further microstructure analysis was conducted on sample S4.

Melting pool boundaries appeared as nose-shape regions, as shown with dotted lines in Fig. 4a. The depth and width of the melting pools ranged from 100 to $200 \mu \mathrm{m}$. The disordered and overlapping boundaries resulted from the alternating $67^{\circ}$ between each layer. It was observed that the solidified melting pool boundaries ended in the previously solidified melting pool boundaries. The closely stacked melting pool boundaries formed good metallurgical bonding between two neighbor layers. Similar structures have been reported in LPBF fabrication of Inconel 625 by other researchers [31].

The cellular and dendritic structures were observed in the melt pool, as shown in Fig. 4b, c. The width of the dendrites was approximately $2 \mu \mathrm{m}$ in Fig. $4 \mathrm{~b}$, and the dendrites preferred to grow across the melting pool boundaries and toward the center of the melting pools, as shown in regions A and B in Fig. 4a. The size of these irregular cellulars varied from 1 to $4 \mu \mathrm{m}$ in Fig. $4 \mathrm{~b}$. Figure $4 \mathrm{~d}$ shows

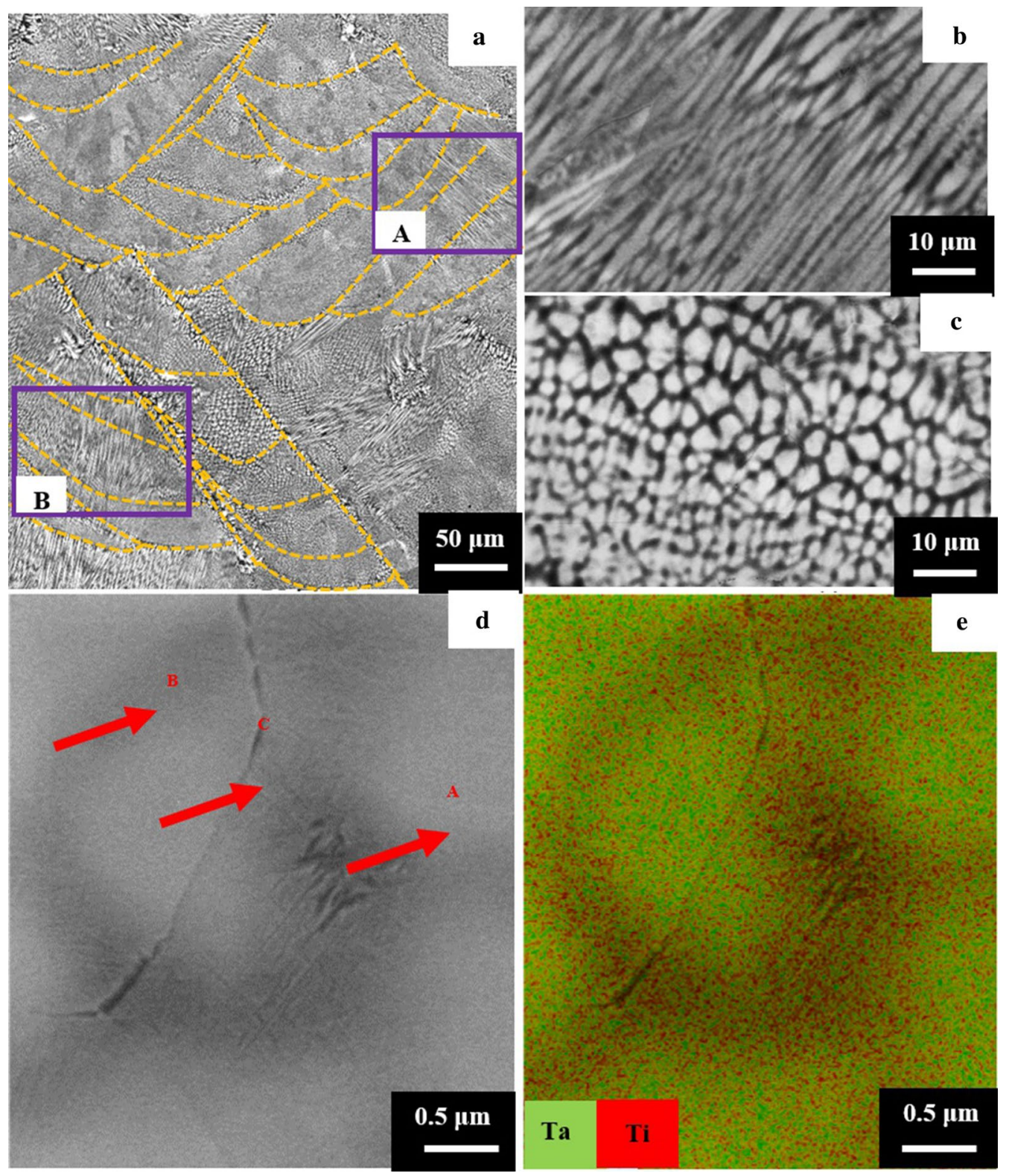

Fig. 4 BSE and EDS micrographs sample 4: a low-magnification microstructure; $\mathbf{b}$ dendrite and $\mathbf{c}$ cellular dendrite morphology; $\mathbf{d}$ high-magnification morphology; e EDS scanning results of cellular dendrites to graph $\mathbf{d}$ 
the high-magnification morphology of one cellular which appears as an irregular shape approximately $1.5 \mu \mathrm{m}$ in diameter. It was observed that the cellular boundary was darker than that of the inside. The corresponding EDS results revealed that the Ti content in the cellular boundary region was higher than the inside, as shown in Fig. 4e and Table 2. This result indicated that microsegregation of the elements occurs in the LPBFed Ti-Ta alloy.

The formation of cellular and dendritic structures is caused by constitutional undercooling [32], which is governed by the criteria required for the onset of constitutional undercooling that are given by: $\frac{G_{\mathrm{L}}}{v}<-\frac{m_{\mathrm{L}} C_{0}(1-k)}{k}=\frac{\Delta T_{0}}{D_{\mathrm{L}}}$, where $k$ is the partition coefficient, $D_{\mathrm{L}}$ is the liquid diffusivity, and $\Delta T_{0}$ is the temperature difference between the equilibrium liquidus and solidus of the alloy of composition $C_{0}$. With increasing $\Delta T_{0}$, constitutional undercooling is more prone to occur. The elements of $\mathrm{Ti}$ and Ta could form continuous solid solution, and melting point of $\mathrm{Ti}\left(1668{ }^{\circ} \mathrm{C}\right)$ was much lower than $\mathrm{Ta}$ $\left(2996{ }^{\circ} \mathrm{C}\right)$. During the rapid solidification of Ti-50 wt\% $\mathrm{Ta}$, the diffusion of Ta element is difficult to achieve equilibrium, and the content of Ta in solid phase was higher at the liquid-solid interface $\left(k_{\mathrm{Ta}}>1\right)$. Hence, Ti was rejected into the solidifying front in the liquid. And the Ti concentration in the liquid increases as the solidification front moves [32]. For conditions of growth where the $G_{\mathrm{L}} / \mathrm{V}$ ratio is only slightly smaller than the ratio of $\Delta T_{0} / D_{\mathrm{L}}$, the interface is cellular in shape, as shown in Fig. 4c. For conditions of growth where the ratio of $G_{\mathrm{L}} / \mathrm{V}$ is much smaller than the ratio of $\Delta T_{0} / D_{\mathrm{L}}$, the interface becomes columnar dendritic in shape [33, 34], as shown in Fig. 4b. Under such circumstances, $\mathrm{Ti}$ is enriched in the solute and results in microsegregation at the end of solidification.

Due to the sharp cooling rates during LPBF induced by the high laser energy density [5], finer cellular and dendritic structures are formed compared to those of regular casting structures [35]. The interplay of the melt pool under the $67^{\circ}$ meander filling strategy [36] and high laser energy [37] would result in instability of the melt pool and a complicated thermal field, which led to the disordered cellular and dendritic structures, as well as the varying grain shapes and sizes. Dendrites (regions A and B in Fig. 4a) and grains (Fig. 5a) are grown in regions with the highest temperature gradient and solidification rate [38]. The cellular and dendritic structures bear a clear intragranular feature, i.e., these regions cannot be interpreted as grains because the adjacent cells have almost the same crystallographic orientation, as shown in Fig. 5a. The shape of the cellular structures could be equiaxed or bar-like depending on the growth direction of the larger columnar grains that contained them, as shown in Fig. 5a.

To investigate the phase constitution of LPBFed Ti-Ta alloy, X-ray diffraction was used, and the result is shown in Fig. 6 . The diffraction peaks of the $\beta$ phase with a body-centered cubic structure and the $\alpha$ " phase with an orthorhombic structure were observed. The familiar $\alpha$ phase or $\alpha^{\prime}$ phase with hexagonal close-packed structure was not detected in

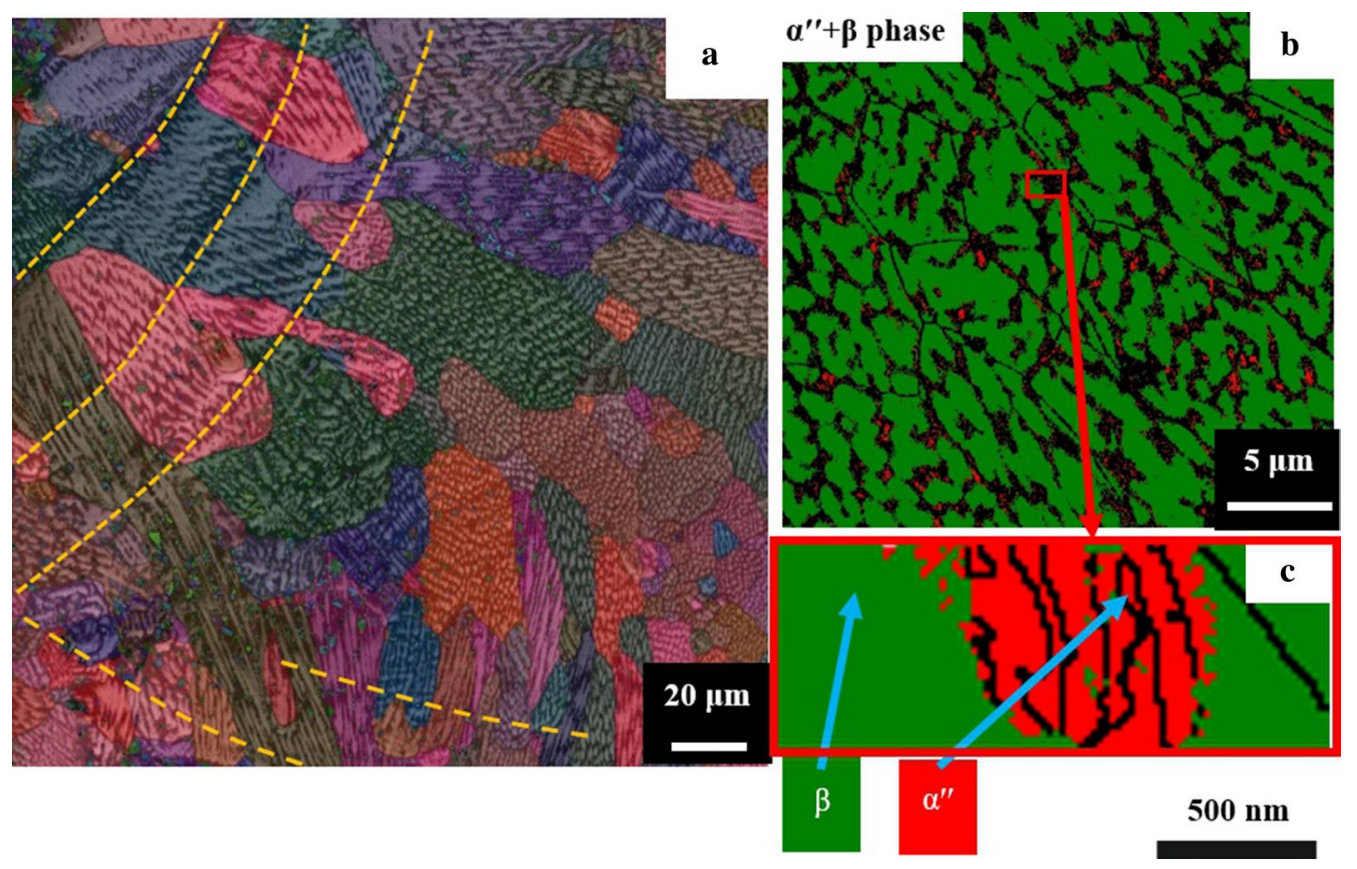

Fig. 5 a Superimposed EBSD map of all Euler and BSE maps for sample 4 specimens; $\mathbf{b}, \mathbf{c}$ EBSD phase map (the green regions represent the $\beta$ phase, and the red regions represent the $\alpha^{\prime \prime}$ phase) 


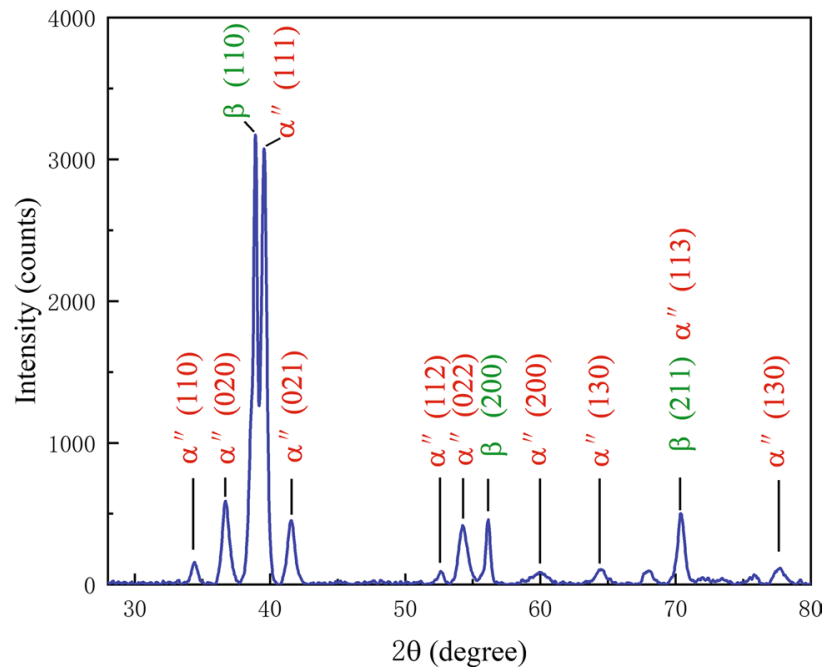

Fig. 6 X-ray diffraction pattern of S4

the diffraction pattern. Accurate phase proportions were difficult to calculate due to the RIR data lacking the $\alpha^{\prime \prime}$ phase.

Figure 5a shows the grains morphology and the dendrite (including cellular) structure. It can be seen that the shape and size of the grains are very different from each other. The cellular and dendrite morphologies are similar within a given grain but are different from grain to grain. The melting pool boundaries can be distinguished as dotted lines. It was seen that the grains and the dendrites preferred to grow perpendicular to the boundaries (toward the center of the melting pool).
Figure $5 \mathrm{~b}$ shows the phase constitutions and distributions, in which the $\beta$ and $\alpha^{\prime \prime}$ phases are shown as green and red, respectively. The lines represent the grain boundaries. It was observed that the $\beta$ phase exists inside the cellular regions and the dendrites. Only a small fraction of $\alpha^{\prime \prime}$ phase was observed, which is inconsistent with the XRD results. It should be noted that several areas with no-index, marked with black, in the interdendritic region were observed. Zooming into these regions resulted in these no-index areas being indexed as $\alpha^{\prime \prime}$ phase, as shown in Fig. 5c. Numerous grain boundaries were found in the $\alpha^{\prime \prime}$ phase areas. The width of the $\alpha^{\prime \prime}$ phase was less than $100 \mathrm{~nm}$.

A similar cellular morphology was observed in the brightfield TEM images, as shown in Fig. 7a, b, which consisted of a cellular boundary (region A) and the region inside the cells (region B). The corresponding selected area diffraction pattern (SADP) of regions A and B is shown in Fig. 7b, c and was identified as $\alpha^{\prime \prime}$ phase and $\beta$ phase, respectively. It was observed that the $\alpha^{\prime \prime}$ phase has a lamellar structure, and the width of the lamellar grains was less than $100 \mathrm{~nm}$. The crystallographic orientations of these $\alpha$ " phase grains were different; thus, some different grains did not appear in the dark-field TEM images shown in Fig. 7d. According to the EDS result (Table 3), the content of Ti is higher in the $\alpha^{\prime \prime}$ phase (region A). Similar to Table 2, the TEM results

Table 3 Element contents of regions A and B in Fig. 7a

\begin{tabular}{lll}
\hline Region & $\mathrm{Ti}(\mathrm{wt} \%)$ & $\mathrm{Ta}(\mathrm{wt} \%)$ \\
\hline A & 53.9 & 46.1 \\
B & 50.5 & 49.5 \\
\hline
\end{tabular}
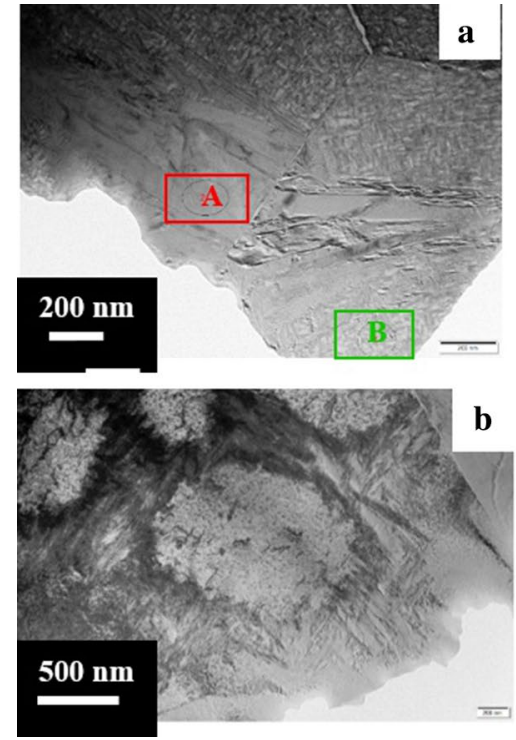
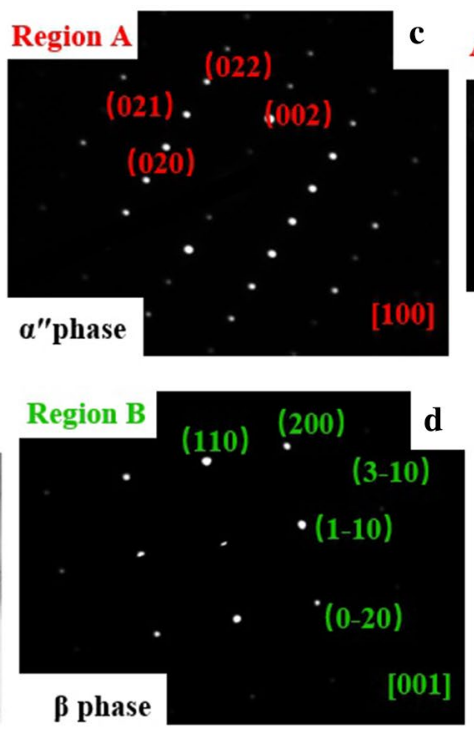

01]

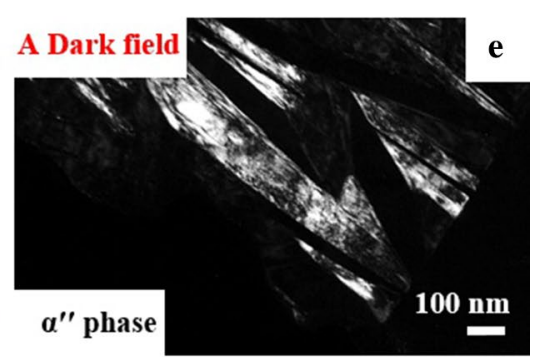

Fig. 7 a, b Bright-field TEM images for sample 4; $\mathbf{c}$ SADP pattern of the $\alpha^{\prime \prime}$ phase; $\mathbf{d}$ SADP pattern of the $\beta$ phase; e dark-field TEM images of the $\alpha^{\prime \prime}$ phase 
confirmed that the titanium experienced microsegregation in the cellular boundary.

The phase composition ( $\alpha^{\prime \prime}$ phase and $\beta$ phase) of LPBFed Ti-50 wt\% Ta alloys was consistent with the previous rapid cooling studies [13, 22, 39]. Martensite ( $\alpha^{\prime}$ and $\left.\alpha^{\prime \prime}\right)$ will form during the process of rapid cooling from the $\beta$ phase field. At a lower tantalum content, the $\beta$ phase will transform to the $\alpha^{\prime}$ phase with decreasing temperature [40]. As tantalum increases, the metastable $\alpha^{\prime \prime}$ phase (the transition phase from $\beta$ phase to $\alpha^{\prime}$ phase) appears during rapid cooling. Excess $\beta$-stabilizing elements will suppress the phase transition completely, and the $\beta$ phase will be retained at room temperature during solidification. For Ti-50 wt $\%$ Ta alloys, the formation of the martensite $\alpha^{\prime}$ phase was totally suppressed, and the martensite $\alpha^{\prime \prime}$ phase and remaining $\beta$ phase were observed. However, the phase distributions of LPBFed Ti-50 wt $\%$ Ta alloys were different from previous rapid cooling studies in which the fine $\alpha^{\prime \prime}$ structure was distributed in a disorderly manner within the $\beta$ grains [13, $22,39]$. In LPBFed Ti-50 wt $\%$ Ta alloys, the $\alpha^{\prime \prime}$ structure was distributed at the boundary of the cellular structures. As mentioned before, the formation of cellular structures in LPBFed Ti-50 wt\% Ta alloys was attributed to the relatively small value of $G_{\mathrm{L}} / \mathrm{V}$. During the LPBF processing of Ti-50 wt $\%$ Ta, the $\beta$ phase was formed first starting with a primary liquid $\rightarrow \beta$ phase reaction and then proceeding to cause element enrichment of the interdendritic liquid, which led to the formation of cellular and dendritic structures. In the areas inside the cellular structures, the tantalum content was high enough to stabilize the $\beta$ phase; however, the $\beta$ phase $\rightarrow \alpha$ " phase transition occurred in the cellular boundary areas where the tantalum content was not high enough to suppress the bcc lattice transition completely but could suppress the $\beta$ phase $\rightarrow \alpha^{\prime}$ phase transition. Thus, the $\beta$ phase inside the cellular and $\alpha^{\prime \prime}$ phase in the cellular boundaries were observed in the LPBFed Ti-50 wt\% Ta alloys.

However, Sing et al. [4] fabricated Ti-Ta parts that consisted of equiaxed $\beta$ grains only via LPBF initially. They found similar cellular morphology, but $\alpha^{\prime \prime}$ phase was not observed in the cellular boundaries. The phase difference might be caused by the different LPBFed equipment and scanning strategies [41]. Besides, this paper investigated the detailed microstructure such as cellular/dendritic features and phase transformation and a new method for controlling microstructures with different phase distributions in a fine cellular structure via laser powder bed fusion.

The Young's modulus of LPBFed Ti-50 wt\% Ta is 97.7 GPa measured by an MTS testing facility (MTS-Landmark 370.25). It is lower than LPBFed Ti6Al4V (120.1 GPa) reported by other researchers [42]. But it is higher than arc melting Ti-50 wt $\%$ Ta (88 GPa) [22] due to slight unmelted Ta particles. It has been reported that Ta addition could enhance the corrosion resistance of LPBFed TiTa alloy
[26]. And the corrosion rate is much lower than pure $\mathrm{Ti}$ in their experiments. Thus, the LPBFed Ti-50 wt\% Ta alloy is promised candidates for biomedical application.

\section{Conclusions}

1. Increasing the exposure time was effective for reducing the unmelted Ta and achieving element homogeneity. Nearly fully dense in situ alloyed Ti-50 wt $\%$ Ta samples were fabricated by laser powder bed fusion.

2. Cellular and dendritic structures were observed in the melt pool due to constitutional undercooling. The cellular and dendritic structures were disordered and fine due to the complicated thermal field and the sharp cooling rate.

3. The LPBFed Ti-50 wt $\%$ Ta alloy consisted of a retained $\beta$ phase and an $\alpha^{\prime \prime}$ phase (orthorhombic martensite). The $\alpha^{\prime \prime}$ phase was observed in the cellular boundaries as a result of the Ti concentration in the solidification front.

Acknowledgements This work was financially supported by the National Natural Science Foundation of China (No. U1605243) and the National Key Program of China (No. 2018YFB1106900).

\section{References}

[1] D. Ao, X. Chu, Y. Yang, S. Lin, J. Gao, Vacuum 148, 230 (2018)

[2] X. Zhou, D. Wang, X. Liu, D. Zhang, S. Qu, J. Ma, G. London, Z. Shen, W. Liu, Acta Mater. 98, 1 (2015)

[3] B. Vrancken, L. Thijs, J.P. Kruth, J. Van Humbeeck, Acta Mater. 68, 150 (2014)

[4] S.L. Sing, W.Y. Yeong, F.E. Wiria, J. Alloys Compd. 660, 461 (2016)

[5] P. Barriobero-Vila, J. Gussone, A. Stark, N. Schell, J. Haubrich, G. Requena, Nat. Commun. 9, 3426 (2018)

[6] W. Xu, E.W. Lui, A. Pateras, M. Qian, M. Brandt, Acta Mater. 125, 390 (2017)

[7] C. Kenel, D. Grolimund, X. Li, E. Panepucci, V.A. Samson, D.F. Sanchez, F. Marone, C. Leinenbach, Sci. Rep. 7, 16358 (2017)

[8] P. Kumar, O. Prakash, U. Ramamurty, Acta Mater. 154, 246 (2018)

[9] M.B.D. BomBač, P. Fajfar, Mater. Geoenviron. 54, 471 (2007)

[10] T. Hanawa, Mater. Sci. Forum 512, 243 (2006)

[11] D. Kuroda, M. Niinomi, M. Morinaga, Mater. Sci. Eng. A 243, 244 (1998)

[12] W.F. Ho, W.K. Chen, S.C. Wu, H.C. Hsu, J. Mater. Sci. Mater. Med. 19, 3179 (2008)

[13] Y.L. Zhou, M. Niinomi, T. Akahori, Mater. Sci. Eng. A 384, 92 (2004)

[14] S. Xu, Y. Liu, C. Yang, H. Zhao, B. Liu, J. Li, M. Song, Mater. Sci. Eng. A 712, 386 (2018)

[15] E.A. Trillo, C. Ortiz, P. Dickerson, R. Villa, S.W. Stafford, L.E. Murr, J. Mater. Sci. Mater. Med. 12, 283 (2001)

[16] Y.L. Zhou, M. Niinomi, Mater. Sci. Eng. C 29, 1061 (2009)

[17] Y. Liu, K. Li, H. Wu, M. Song, W. Wang, N. Li, H. Tang, J. Mech. Behav. Biomed. Mater. 51, 302 (2015) 
[18] J. Kesteven, M.B. Kannan, R. Walter, H. Khakbaz, H.C. Choe, Mater. Sci. Eng. C 46, 226 (2015)

[19] M. Niinomi, Metall. Mater. Trans. A 33, 477 (2002)

[20] S. Banerjee, P. Mukhopadhyay, Phase Transformations: Examples from Titanium and Zirconium Alloys (Elsevier, Amsterdam, 2010)

[21] E. Zhang, S. Zeng, X. Zeng, Q. Li, Acta Metall. Sin. (Engl. Lett.) 8, 130 (2009)

[22] Y.L. Zhou, M. Niinomi, T. Akahori, Mater. Sci. Eng. A 371, 283 (2004)

[23] Y.L. Zhou, M. Niinomi, J. Alloys Compd. 466, 535 (2008)

[24] S.L. Sing, F.E. Wiria, W.Y. Yeong, Int. J. Refract. Met. Hard Mater. 77, 120 (2018)

[25] S.L. Sing, F.E. Wiria, W.Y. Yeong, Robot. Comput. Integr. Manuf. 49, 170 (2018)

[26] D. Zhao, C. Han, Y. Li, J. Li, K. Zhou, Q. Wei, J. Liu, Y. Shi, J. Alloys Compd. 804, 288 (2019)

[27] I. Yadroitsev, P. Krakhmalev, I. Yadroitsava, J. Met. 69, 2725 (2017)

[28] P. Fischer, V. Romano, H.P. Weber, N.P. Karapatis, E. Boillat, R. Glardon, Acta Mater. 51, 1651 (2003)

[29] L.Z. Wang, W.H. Wei, Acta Metall. Sin. (Engl. Lett.) 31, 807 (2018)

[30] Y.L. Guo, L.N. Jia, B. Kong, Y.L. Huang, H. Zhang, Acta Metall. Sin. (Engl. Lett.) 31, 477 (2017)
[31] I. Yadroitsev, L. Thivillon, P. Bertrand, I. Smurov, Appl. Surf. Sci. 254, 980 (2007)

[32] M.C. Flemings, Metall. Trans. 5, 2121 (1974)

[33] Y.F. Li, C. Li, J. Wu, H.J. Li, Y.C. Liu, H.P. Wang, Acta Metall. Sin. (Engl. Lett.) 32, 764 (2018)

[34] A. Barros, C. Cruz, A.P. Silva, N. Cheung, A. Garcia, O. Rocha, A. Moreira, Acta Metall. Sin. (Engl. Lett.) 32, 695 (2018)

[35] H. Schwab, F. Palm, U. Kühn, J. Eckert, Mater. Des. 105, 75 (2016)

[36] Z. Wang, K. Guan, M. Gao, X. Li, X. Chen, X. Zeng, J. Alloys Compd. 513, 518 (2012)

[37] L. Thijs, F. Verhaeghe, T. Craeghs, J. Humbeeck, J. Kruth, Acta Mater. 58, 3303 (2010)

[38] K.G. Prashanth, S. Scudino, H.J. Klauss, K.B. Surreddi, L. Löber, Z. Wang, A.K. Chaubey, U. Kühn, J. Eckert, Mater. Sci. Eng. A 590, 153 (2014)

[39] K.A. Bywater, J.W. Christian, Philos. Mag. 25, 1249 (1972)

[40] E.H. Froes, Titanium: Physical Metallurgy, Processing, and Applications (ASM International, Cleveland, 2015)

[41] X. Wang, F. Lv, L.D. Shen, H.X. Liang, D.Q. Xie, Z.J. Tian, Acta Metall. Sin. (Engl. Lett.) 32, 1173 (2018)

[42] X. Yan, S. Yin, C. Chen, C. Huang, R. Bolot, R. Lupoi, M. Kuang, W. Ma, C. Coddet, H. Liao, M. Liu, J. Alloys Compd. 764, 1056 (2018) 